\title{
Avaliação da Toxicidade do Endosulfan Sulfato sobre Juvenis de Danio Rerio (Cypriniformes, Cyprinidae)
}

\author{
R. Fracácio, ${ }^{1 *}$ A. F. Campagna, ${ }^{1}$ B. K. Rodrigues, ${ }^{1}$ N. F. Verani ${ }^{1}$ \& E. L. G. Espíndola ${ }^{2}$ \\ 1 Departamento de Hidrobiologia, Universidade Federal de São Carlos, \\ Rod. Washington Luis (SP-310), km 235, CEP 13565-905, São Carlos, SP \\ 2 Departamento de Hidráulica e Saneamento, Universidade de São Paulo, \\ Av. Trabalhador São-carlense, 400, CEP 13560-600, São Carlos, SP
}

(Received December 10, 2007; Accepted September 25, 2008)

\begin{abstract}
RESUMO
O objetivo do presente trabalho foi avaliar a toxicidade do endosulfan sulfato (forma mais persistente do produto) sobre juvenis de Danio rerio, considerando-se as mortalidades em 96 horas de exposição, e a sobrevivência e alterações histológicas de brânquias em testes de toxicidade crônica parcial (duração de 7 dias) em concentrações de $0,003 \mu \mathrm{g} \mathrm{L}^{-1}, 0,3 \mu \mathrm{g} \mathrm{L} \mathrm{L}^{-1} \mathrm{e} 3,0 \mu \mathrm{g}$ $\mathrm{L}^{-1}$, sendo a concentração intermediária detectada em rios da bacia hidrográfica do Alto Jacaré-Guaçu (São Paulo, Brasil). Em relação à mortalidade, obteve-se uma $\mathrm{CL}_{50}, 96 \mathrm{~h}$ de $7,29 \mu \mathrm{g} \mathrm{L}{ }^{-1}$. Considerando-se a sobrevivência, somente a maior concen-tração do produto $\left(3 \propto \mathrm{g} \mathrm{L}^{-1}\right)$ foi tóxica aos organismos-teste. Entretanto, os resultados obtidos nas análises histológicas qualitativas, em todas as concentrações, indicaram modificações teciduais do epitélio branquial representadas pela hiperplasia, junções entre algumas lamelas secundárias, além do espessamento no ápice de algumas lamelas secundárias, as quais são alterações que comprometem as trocas gasosas e, conseqüentemente, prejudicam as funções vitais do sistema biológico. As alterações obtidas no presente estudo são de primeiro e segundo graus, as quais podem ser reversíveis com a melhoria das condições químicas, ou, então, podem progredir para um estágio de terceiro grau, o qual é irreversível, culminando com a morte dos indivíduos. Estes resultados confirmam a importância da histologia como ferramenta de avaliação da toxicidade de produtos químicos e evidenciam que o endosulfan sulfato é tóxico para essa espécie de peixe em concentrações registradas no ambiente aquático acima descrito.
\end{abstract}

Palavras-chave: Danio rerio, endosulfan sulfato, histologia, toxicidade.

\section{ABSTRACT \\ Evaluation of toxicity endosulfan sulfate on Danio rerio juvenilis (Cypriniformes, Cyprinidae)}

The objective of this work was to evaluate the toxicity of endosulfan sulfate toxicity (the most persistent form) in Danio rerio juveniles, considering the mortalities, in exposition of 96 hours, and survive and microscopic gill morphology in toxicity test partial chronic (7 days) in concentrations of $0.003 \mu \mathrm{g} \mathrm{L}^{-1}, 0.3 \mu \mathrm{g} \mathrm{L}^{-1}$ and $3.0 \mu \mathrm{g} \mathrm{L}^{-1}$, near the levels detected in the upper Jacaré-Guaçu river basin (São Paulo, Brazil). The result of the mortality was detected CL50, $96 \mathrm{~h}$ of the $7.29 \mu \mathrm{g} \mathrm{L}^{-1}$. Considering the survival, only the higher concentration $\left(3 \mu \mathrm{g} \mathrm{L}^{-1}\right)$ promoted toxicity to the test organisms. However, the histological analyses revealed gill changes such as hyperplasia, fusion between some secondary lamellae and thickening of the apex of some secondary lamellae. These alterations compromised the gases exchange and consequently the vital functions of the biological system. The obtained alterations were of first and second level, but these changes can either to return to normal conditions when chemistry conditions improve, or to progress to a third stage degree, which are irreversible, provoking the death of the individual. This results prove the importance of the histology as a tool to evaluate toxicity of chemical products, giving evidences that endosulfan sulfate is toxic to this fish specie at concentrations found in aquatic environments.

Key words: Danio rerio, endosulfan sulfate, histology, toxicity.

*Corresponding author: Renata Fracácio, e-mail: rfracacio@uol.com.br. 


\section{INTRODUÇÃO}

Os pesticidas representam um grupo químico único por serem ministrados intencionalmente no ambiente para destruir ou controlar formas de vida indesejadas. No entanto, repre-sentam um risco por não atingirem somente os organismos-alvo. Além disso, muitos persistem no ambiente por longos períodos de tempo (Dores \& Freire, 1999).

Dentre os inseticidas orgânicos sintéticos, os mais persistentes no meio ambiente são os organoclorados. Isto porque têm elevada estabilidade química e baixa degradabilidade, são insolúveis em água e apresenta alta capacidade de ligação com a gordura animal. Portanto, são estáveis no meio físiológico (Rand \& Petrocelli, 1985; Buratini \& Brandelli, 2006).

Diferentes estudos têm enfocado a toxicidade de organoclorados em peixes de água doce, demonstrando efeitos como: hiperglicemia e diminuição de conteúdos protéicos no fígado e no músculo; hipocalcemia; hiperfosfatemia e danos epiteliais aos tecidos respiratórios (Singh et al., 1996); disfunções renais (Rashatwar \& Ilyas, 1984); diminuição nas taxas de sobrevivência e de eclosão de ovos (Roex et al., 2002); alterações no cres-cimento e reprodução (Gormley \& Teather, 2003); desordem neuromuscular; má formação esquelética e tremores; bioacu-mulação em diferentes órgãos e efeitos teratogênicos; dentre outros. Esses efeitos são decorrentes de exposições a concen-trações subletais, comumente encontradas em ambientes natu-rais (Owens \& Baer, 2000).

Por essas razões, diversos países proibiram o uso desses grupos químicos. No Brasil, em 1985, vários pesticidas tiveram o uso proibido em virtude de suas propriedades carcinogênicas, e alguns passaram a ter uso limitado pela Portaria 329 de 02/ 09/1985, em razão de seu caráter cumulativo.

$\mathrm{O}$ endosulfan, nome comercial Thiodan, é o único com-posto organoclorado cujo uso ainda é permitido em função de sua baixa persistência e bioacumulação. No Brasil, seu uso é destinado às plantações de algodão e ao combate à "broca da cana" (CBH-Mogi, 1999).

Esse organoclorado é constituído por dois isômeros, o $\alpha$ e o $\beta$, os quais apresentam meia-vida de aproximadamente 24 h. Ao sofrerem degradação, ambos os isômeros permanecem na forma de endosulfan sulfato, o qual é mais persistente na água com meia-vida de cinco a sete dias, dependendo das condições de temperatura e $\mathrm{pH}$ do ambiente (Antonious \& Bier, 1997).

Em rios brasileiros, têm sido detectadas concentrações de endosulfan total (sulfato $+\alpha+\beta$ ) acima da permitida pela legislação brasileira (CONAMA 357/05) para a proteção da vida aquática de $\left(0,056 \propto \mathrm{g} \mathrm{L}^{-1}\right)$. Como exemplo, PélaezRodriguêz (2001) e Campagna et. al. (2007) detectaram concentrações de endosulfan sulfato que variaram de $0,014 \propto \mathrm{g}$ $\mathrm{L}^{-1}$ a $0,113 \propto \mathrm{g} \mathrm{L}^{-1}$, na bacia do Alto Jacaré-Guaçu (São Paulo, Brasil), em áreas próximas a monoculturas de cana-de-açúcar. Entretanto, os efeitos subletais desse organoclorado são pouco conhecidos e relatados na literatura.

Diante do exposto, o objetivo deste trabalho foi avaliar a toxicidade do endosulfan sulfato (forma mais persistente do produto) em um curto período de exposição ( 96 h), considerando-se a mortalidade, bem como averiguar os efeitos das concentrações subletais em tempo maior de exposição (teste crônico parcial de 7 dias), considerando-se as respostas de sobrevivência e alterações histológicas qualitativas do epitélio branquial de Danio rerio na fase juvenil. Para a realização deste trabalho, as concentrações de endosulfan sulfato detecta-das em rios da Bacia do Alto Jacaré-Guaçu, a qual está inserida em áreas de monoculturas de cana-de-açúcar, bem como aquelas permitidas pela legislação brasileira para a proteção da vida aquática, foram consideradas.

\section{MATERIAIS E MÉTODOS}

\section{Organismo-teste e manutenção}

Utilizou-se a espécie Danio rerio na fase juvenil como organismo-teste, por ser de fácil manutenção em laboratório, aquisição comercial a baixos custos e por ter metodologia padronizada para testes de toxicidade aguda (ABNT, 2003) e crônica de curta duração para organismos embriolarvais (ABNT, 2007). A fase juvenil foi identificada de acordo com o tamanho dos organismos, seguindo-se a correlação comprimento com a maturação gonadal estabelecida por Maack \& Segner (2003). Os organismos utilizados apresentaram o comprimento médio de $1,1 \mathrm{~cm}( \pm 0,016)$.

Os organismos-teste foram obtidos comercialmente e mantidos no laboratório em aquários de 25 litros, com metade do volume composto pela água do próprio cultivo e outra parte com água de manutenção ABNT (2003). A substituição de 1/3 da água por água reconstituída foi realizada a cada $24 \mathrm{~h}$. A aeração foi mantida constante e os valores de $\mathrm{pH}$, condutividade e dureza foram correspondentes a 7,4, 180 $\propto \mathrm{S} / \mathrm{cm}^{-1}$ e $46 \mathrm{mg} \mathrm{CaCO} 3 \mathrm{~L}^{-1}$, respectivamente. A alimentação ministrada foi ração comercial Tetramim $\rightarrow$, a qual foi suspensa $24 \mathrm{~h}$ antes da rea-lização dos testes de toxicidade.

\section{Teste de sensibilidade}

A sensibilidade de cada lote de organismos foi avaliada por meio de uma substância de referência, o dicromato de potássio $\left(\mathrm{K}_{2} \mathrm{Cr}_{2} \mathrm{O}_{7}\right)$. Para isto, testes de sensibilidade foram realizados pela exposição dos organismos-teste a diferentes concentrações de dicromato de potássio, buscando-se encontrar a concentração letal a $50 \%$ dos organismos $\left(\mathrm{CL}_{50}, 96 \mathrm{~h}\right)$, em regime estático (ABNT, 2003).

\section{Testes de toxicidade com o organoclorado endosulfan sulfato}

Foi obtido o padrão puro de endosulfan sulfato, P.A: 16823-0048 na empresa SUPELCO. Por ser insolúvel em água, foi utilizada acetona P.A como veículo de dispersão desse composto na água reconstituída dos testes (SEMA, 1988).

Foram realizados testes de toxicidade aguda semiestáticos, com trocas do meio a cada $24 \mathrm{~h}$ e duração de $96 \mathrm{~h}$, seguindo-se a metodologia descrita em ABNT (2003). A faixa de sensibilidade foi determinada utilizando-se os valores de $\mathrm{CL}(\mathrm{I}) 50,96 \mathrm{~h}$, seguindo-se as recomendações da USEPA (1985). O cálculo desses valores foi realizado com o auxílio do programa computacional 
Trimmed Spearman-Karber (Hamilton et al., 1977). As concentrações estabelecidas foram de $3 \propto \mathrm{g} \mathrm{L}^{-1} ; 4,5 \propto \mathrm{g} \mathrm{L}^{-1}$; $6,75 \propto \mathrm{g} \mathrm{L}^{-1}$; e $10,25 \propto \mathrm{g} \mathrm{L}^{-1}$. Para cada concentração, foram montadas 3 réplicas com 10 organismos-teste em cada uma. No total, foram feitas 7 repetições do teste. Foram montados controles com água reconstituída e controle contendo a concentração máxima do solvente acetona, utilizado em testes de toxicidade $\left(100 \propto 1 \mathrm{~L}^{-1}\right)($ SEMA, 1988).

Posteriormente, foram montados testes de toxicidade crônica parciais de sete dias, em regime semiestático, com trocas do meio a cada $24 \mathrm{~h}$. Seguiu-se a metodologia descrita em ABNT (2007), modificando-se apenas a fase de vida do organismo-teste de embriolarval para juvenil. Foram montadas quatro réplicas por concentração com 10 organismos em cada, con-siderando-se as concentrações estabelecidas $\left(0,03 \propto \mathrm{g} \mathrm{L}^{-1}\right.$; $0,3 \propto \mathrm{g} \mathrm{L}^{-1}$; e $3 \propto \mathrm{g} \mathrm{L}^{-1}$ ), as quais foram determinadas a partir da menor concentração de efeito não observado (CENO) dos testes agudos, dividindo-se esse valor por 10, e assim sucessivamente, para determinar as duas concentrações abaixo da CENO. Desta maneira, a menor concentração detectada no ambiente natural pôde ser simulada em laboratório com os organismos-teste. Além do meio com as diferentes concentrações da substância tóxica, foram montados controles com água reconstituída e controles contendo a concentração máxima de acetona utilizada nos testes, de $100 \propto \mathrm{L} \mathrm{L}^{-1}$, concentração máxima recomendada na norma SEMA (1988). Foram feitas 3 repetições dos testes crônicos parciais.

A mortalidade dos organismos-teste e as variáveis $\mathrm{pH}$, condutividade e dureza da água foram monitoradas a cada $24 \mathrm{~h}$. Os testes foram montados em béqueres de vidro, com capacidade para $1 \mathrm{~L}$, adicionando-se $500 \mathrm{~mL}$ de soluções-teste por réplica. Os testes foram mantidos em sala climatizada com fotoperíodo $12 \mathrm{~h}: 12 \mathrm{~h}$ claro/escuro, temperatura constante de $26^{\circ} \mathrm{C} \pm 1{ }^{\circ} \mathrm{C}$ e aeração constante. Durante os testes e $24 \mathrm{~h}$ antes da montagem dos mesmos, foi suspensa a alimentação.

Ao final dos testes, os resíduos foram armazenados em recipientes apropriados e encaminhados à estação de tratamento de resíduos da Universidade de São Paulo, São Carlos, SP.

\section{Tratamento dos resultados obtidos nos testes de toxicidade crônica parcial}

Sobrevivência: Foi utilizado o teste exato de Fisher para comparar as proporções de organismos vivos e mortos do controle com as detectadas em cada concentração dos testes crônicos parciais para avaliar a significância dos resultados (Ayres et al., 2001).

Análise histológica de brânquias: após a realização das biometrias, cinco organismos-teste de cada concentração foram fixados em líquido de Bouin por 18 horas e lavados em água corrente por 24 horas, permanecendo em álcool $70 \%$ até o momento da desidratação. Seguiu-se o procedimento recomendado de desidratação e diafanização para a inclusão dos organismos em parafina (Tolossa et al., 2003). Foram realizados cortes seriados de 5 a $7 \propto \mathrm{m}$ de espessura com navalha de aço em micrótomo (Micron HM 340E). A coloração utilizada foi hematoxilina-eosina (HE), e a avaliação qualitativa dos tecidos branquiais foi feita por meio de microscópio de luz. As alterações foram identificadas seguindo-se Takashima \& Hibia (1995) e a classificação proposta por Poleksic \& Mitrovic-Tutundizc (1994), relatadas na Tabela 1.

\section{RESULTADOS}

A partir dos testes de toxicidade aguda com endosulfan sulfato, obteve-se o valor médio da $\mathrm{CL}_{50}, 96 \mathrm{~h}$ de $7,29 \propto \mathrm{g} \mathrm{L}^{-1}$. A maior concentração de efeito não observado, estatisticamente significativo (CENO), foi de $3 \propto \mathrm{g} \mathrm{L}^{-1}$, sendo, portanto, o valor máximo utilizado nos testes de toxicidade crônica parcial, a partir do qual se obtiveram as concentrações de $0,3 \propto \mathrm{g} \mathrm{L}^{-1}$ e $0,003 \propto \mathrm{g} \mathrm{L}^{-1}$, utilizando-se o fator 10 para as diluições.

Nos testes de toxicidade crônica parcial, as menores porcentagens de sobrevivência foram detectadas na maior concentração $\left(3 \mu \mathrm{g} \mathrm{L}^{-1}\right)$, nas três repetições realizadas, com valores correspondentes a $77,5 \%, 75 \%$ e $67,5 \%$, nos testes número 1,2 e 3 , respectivamente (Tabela 2). Essas porcentagens de sobrevivência indicaram a toxicidade da concentração de $3 \propto \mathrm{g} \mathrm{L}^{-1}$, segundo os resultados obtidos no teste estatístico exato de Fisher (Tabela 2). Já nas concentrações de $0,003 \propto \mathrm{g} \mathrm{L} \mathrm{L}^{-1}$ e $0,3 \propto \mathrm{g} \mathrm{L} \mathrm{L}^{-1}$, as menores porcentagens de sobrevivência (de $87,5 \%$ e de $90 \%$, respectivamente, no teste 3 ) não demonstraram a toxicidade aos organismos-teste (Tabela 2).

Com relação às condições dos testes realizados, observou-se pouca variação quanto ao $\mathrm{pH}$, o qual, considerando-se as diferentes concentrações e repetições, permaneceu acima de 7,2 e abaixo de 7,9. Em relação à condutividade, pode-se notar um aumento dos valores em $24 \mathrm{~h}$ para todas as concentrações, nas diferentes repetições. $\mathrm{O}$ valor máximo registrado para condutividade foi de $334 \propto \mathrm{S} \mathrm{cm} \mathrm{cm}^{-1}$, nos testes 2 e 3 , na concentração de $0,03 \propto \mathrm{g} \quad \mathrm{L}^{-1}$. Os valores de dureza permaneceram entre $32 \mathrm{mg} \mathrm{CaCO}_{3} \mathrm{~L}^{-1}$ e $52 \mathrm{mg} \mathrm{CaCO}_{3} \mathrm{~L}^{-1}$, também considerando-se todas as concentrações e repetições.

\section{Avaliação histológica das brânquias}

Os organismos do controle (Figura 1A) e os organismos do controle mais acetona (Figura 1B) apresentaram a estrutura branquial bem definida, com o tecido epitelial justaposto às lamelas primárias e secundárias (LS) e com os espaços entre as lamelas secundárias bastante amplos e nítidos.

Nos organismos submetidos aos testes de toxicidade na concentração de $0,03 \propto \mathrm{g} \mathrm{L}^{-1}$, verificou-se o espessamento de algumas lamelas secundárias como consequência da proliferação celular, havendo pequena redução entre os espaços interlamelares, os quais ainda se apresentam satisfatórios para a realização das trocas gasosas (Figura 1C).

Os efeitos no epitélio branquial dos organismos expostos a $0,3 \propto \mathrm{g} \mathrm{L}^{-1}$ de endosulfan sulfato foram a hiperplasia do epitélio respiratório, com consequente junção entre algumas lamelas secundárias, obstruindo completamente, nesses locais, os espaços para as trocas gasosas. Foi verificado, também, o espessamento do ápice de algumas lamelas secundárias e a consequente desorganização do epitélio respiratório (Figura 1D). 
Tabela 1 - Principais alterações branquiais e estágio de cada lesão correspondente, segundo Poleksic \& Mitrovic-Tutundizc (1994).

\begin{tabular}{cc}
\hline Lesões branquiais & Estágio \\
\hline Hipertrofia do epitélio respiratório & I \\
Desprendimento das células do epitélio & I \\
Infiltração de leucócitos no epitélio branquial & I \\
Adelgamento do epitélio & I \\
Hiperplasia das células epiteliais & I \\
Hiperplasia na base das lamelas secundárias & I \\
Hiperplasias irregulares nas células epiteliais & I \\
Fusão no ápice das lamelas secundárias & I \\
Diminuição no tamanho das lamelas secundárias & I \\
Hiperplasia e hipertrofia das células mucosas & I \\
Esvaziamento com desprendimento das células mucosas & I \\
Hiperplasia e hipertrofia das células cloreto & I \\
Presença de células cloreto nas lamelas secundárias & I \\
Espessamento dos vasos sanguíneos & I \\
Parasitas nas brânquias & I \\
Ruptura e desprendimento do epitélio branquial & II \\
Fusão completa de todas as lamelas secundárias & II \\
Hemorragias com a ruptura dos tecidos & II \\
Adensamento tecidual em decorrência da proliferação celular & II \\
\hline III \\
III \\
\hline
\end{tabular}

Tabela 2 - Sobrevivência dos organismos-teste, em porcentagem, nos três testes de toxicidade crônica parciais com endosulfan sulfato.

\begin{tabular}{|c|c|c|c|c|c|}
\hline \multirow[b]{2}{*}{ Testes } & \multicolumn{5}{|c|}{ Porcentagens de sobrevivência em sete dias de exposição } \\
\hline & Controle & Controle + acetona & $0,003 \propto g^{-}$ & $0,3 \propto \mathrm{g} \mathrm{L}^{-1}$ & $3 \propto g ~ L^{-1}$ \\
\hline Teste 1 & $100 \%$ & $90 \%$ & $97,5 \%$ & $97,5 \%$ & $77,5 \%(*)$ \\
\hline Teste 2 & $95 \%$ & $97,5 \%$ & $95 \%$ & $95 \%$ & $75 \%(*)$ \\
\hline Teste 3 & $95 \%$ & $97,5 \%$ & $87,5 \%$ & $90 \%$ & $67,5 \%(*)$ \\
\hline
\end{tabular}

$(*) \mathrm{p} \leq 0,05$.

As alterações histológicas detectadas nos organismos da concentração de $3,0 \propto \mathrm{g} \mathrm{L} \mathrm{L}^{-1}$ foram as mesmas das concentrações acima citadas, porém, com maior intensidade. Foi verificada intensa proliferação celular na maioria das lamelas secundárias, resultando na junção de várias delas. Essa alteração implicou a vedação dos espaços para as trocas gasosas em grande parte das brânquias (Figura 1E).

\section{Discussão dos testes de toxicidade com endosulfan sulfato}

De acordo com Jonsson et al. (1992), o endosulfan é um inseticida organoclorado que tem se mostrado altamente tóxico para peixes, com toxicidade da ordem de partes por bilhão. Sunderan et al. (1992) registraram valores médios de CL $_{50} 96$ h, que variaram desde $0,014 \propto \mathrm{g} \mathrm{L}^{-1}$ (Rasbora heteromorpha) a $14 \propto \mathrm{g} \mathrm{L}^{-1}$ (Clarias batrachus). No presente trabalho, esse valor, para os juvenis de D. rerio, foi de 7,29 $\propto \mathrm{g} \mathrm{L}^{-1}$, indicando sensibilidade média ao produto, comparando-se com outras espécies consideradas pelo referido autor, o que justificou a utilização dessa espécie na avaliação da toxicidade do produto.

Os testes de toxicidade, obtidos na literatura, são muito diversificados quanto ao tipo (estático, semiestático ou fluxo contínuo), condições do meio ( $\mathrm{pH}$, dureza, temperatura, dentre 
outros fatores), produto utilizado para a montagem dos testes [grade técnica, produto comercial (thiodan, endosulfan- $\alpha$, endosulfan- $\beta$ e endosulfan sulfato), dentre outros] e duração dos testes ( $24 \mathrm{~h}$ a $96 \mathrm{~h}$ ), o mesmo ocorrendo em relação aos organismos- teste e as fases do ciclo de vida. Essas variações interferem na toxicidade e dificultam a comparação dos dados e afirmações a respeito da sensibilidade da espécie.

No presente estudo, utilizou-se o endosulfan sulfato padrão supelco, caracterizado por apresentar pureza de 99,9\%. Esse produto é volátil a uma temperatura de $45^{\circ} \mathrm{C}$ ou superior e apresenta meia-vida de aproximadamente 7 dias. Portanto, é considerado mais estável que os produtos técnicos e comerciais, cujo tempo de vida é praticamente a metade, além de conterem outras misturas que não apenas o isômero sulfato.

Nessas condições, a toxicidade do endosulfan sulfato, considerando-se a sobrevivência, foi obtida somente na maior concentração $\left(3 \propto \mathrm{g} \mathrm{L}^{-1}\right)$. Assim, pode-se afirmar que há relação direta da toxicidade do produto com o tempo de exposição dos organismos-teste, uma vez que em 96 horas de exposição não houve toxicidade quanto à resposta de mortalidade de juvenis de $D$. rerio na mesma concentração. Já em 7 dias, além de a sobrevivência indicar o efeito nocivo do endosulfan sulfato sobre os peixes, as análises histológicas corroboraram esses dados. Nos organismos sobreviventes, foram identificadas alterações de segundo grau no epitélio branquial, como a junção de várias lamelas secundárias, o que reduz o espaço para as trocas gasosas. Segundo Polecsic-Mitrovic (1994), as alterações de segundo grau tendem a progredir para alterações de terceiro grau, irreversíveis e letais, caso as condições desfavoráveis permaneçam no ambiente.

Rao \& Murty (1982) relataram que, desde 1979, têm sido reportados dados sobre a toxicidade do endosulfan a organismos aquáticos de água doce e salgada, com efeitos agudos e subletais diversificados, de acordo com a espécie, em virtude das diferentes capacidades de detoxificar o produto. Os autores verificaram que, dentre os resíduos, o endosulfan sulfato e o endosulfan- $\alpha$ apresentam elevada capacidade de se ligarem aos tecido epiteliais, cerebrais, branquiais, hepáticos, renais e musculares, comprometendo o funcionamento dos mesmos.

A toxicidade do produto em questão também foi obtida para as concentrações de $0,03 \mu \mathrm{g} \mathrm{L}^{-1}$ e de $0,3 \mu \mathrm{g} \mathrm{L}^{-1}$ de endosulfan sulfato se considerarmos as análises histológicas, o que não foi demonstrado nas respostas de sobrevivência para as referidas concentrações. Essas concentrações aproximam-se das detecta-das no ambiente real (valor mínimo de $0,014 \propto \mathrm{g} \mathrm{L}^{-1} \mathrm{e}$ máximo de $0,113 \propto \mathrm{g} \mathrm{L}^{-1}$, segundo literatura). Em 7 dias de exposição, a proliferação celular e a desorganização do epitélio respiratório foram indicativas de que houve uma resposta biológica diante da nova condição do ambiente.

Diante do exposto, os resultados demonstraram que a avaliação histológica foi uma ferramenta complementar que contribuiu com a interpretação da toxicidade do organoclorado endosulfan sulfato em peixes. Nem sempre a avaliação da sobrevivência isoladamente é o suficiente para avaliar o poten- cial tóxico de um produto sobre um alvo biológico. Antes da falência dos sistemas biológicos, atua o agente químico, que acarreta a desorganização e a desestruturação celular e, consequentemente, a perda de suas funções, o que pode vir a comprometer não só a sobrevivência do indivíduo, mas a perpetuação da espécie.

A hiperplasia, a junção de várias lamelas secundárias e o espessamento no ápice e na base das lamelas secundárias detectados nos organismos-teste no presente estudo são alterações de primeiro e segundo grau, de acordo com a classi-ficação de Poleksic \& Mitrovic-Tutundizc (1994) (Tabela 1). Estas podem ser reversíveis, caso as condições adversas sejam minimizadas, mas também podem continuar a progredir, alcan-çando um estágio de terceiro grau, como a necrose e fibrose, irreversíveis, em que o organismo morre por asfixia, derivada do comprometimento e da obstrução da área necessária às trocas gasosas.

Domitrovic (2001) observou que, em 96 horas de exposição à concentração de $5 \propto \mathrm{g} \mathrm{L}^{-1}$ de Thiodan (endosulfan comercial), as brânquias de Aequidens portalegrensis apresentaram dilatação dos vasos sanguíneos, edemas subepiteliais e he-morragias, todas alterações de segundo grau. No presente trabalho, em organismos expostos por período de tempo mais prolongado à concentração de $3 \propto \mathrm{g} \mathrm{L}^{-1}$ de endosulfan sulfato, verificaram-se outras alterações de segundo grau, baseadas na classificação proposta por Poleksic \& Mitrovic-Tutundizc (1994). Isso demonstra a sensibilidade de cada espécie ao produto químico, bem como o modo de atuação das diferentes formas do produto.

Jonsson et. al (1992), expondo adultos de D. rerio e de Hyphessobricon bifasciatus durante 21 dias a $0,4 \propto \mathrm{g} \mathrm{L}^{-1}$ de endosulfan técnico, em regime semiestático (renovação a cada $24 \mathrm{~h}$ ), observaram, para as duas espécies, dilatação dos vasos sanguíneos no seio venoso central (da lamela primária) para $D$. rerio e focos de necrose epitelial, que são alterações de terceiro grau e irreversíveis. Portanto, no presente trabalho, o tempo de exposição 3 vezes menor não foi suficiente para causar efeitos subletais de terceiro grau, como os autores detectaram para $D$. rerio. No entanto, é preciso considerar que, em ambientes naturais, tais condições podem ser decorrência da contínua exposição a concentrações subletais.

Se considerarmos que, na Bacia do Alto Jacaré-Guaçu, a amplitude de concentração de endosulfan sulfato é de $0,014 \propto \mathrm{g}$ $\mathrm{L}^{-1}$ a $0,113 \propto \mathrm{g} \mathrm{L}^{-1}$, e que o máximo permitido para a proteção da vida aquática é de $0,056 \propto \mathrm{g} \mathrm{L}^{-1}$ de endosulfan total (sulfato, alfa e beta), podemos concluir que, embora exista a sensibilidade seletiva de cada espécie a produtos químicos, o endosulfan sulfato apresenta grande risco de potencial tóxico para os organismos presentes no ambiente em questão.

A realidade dos ambientes aquáticos naturais é a perma-nência de poluentes em concentrações subletais, as quais devem ter seu efeito tóxico avaliado sobre as espécies pre-sentes, uma vez que podem comprometer a viabilidade das mesmas. 

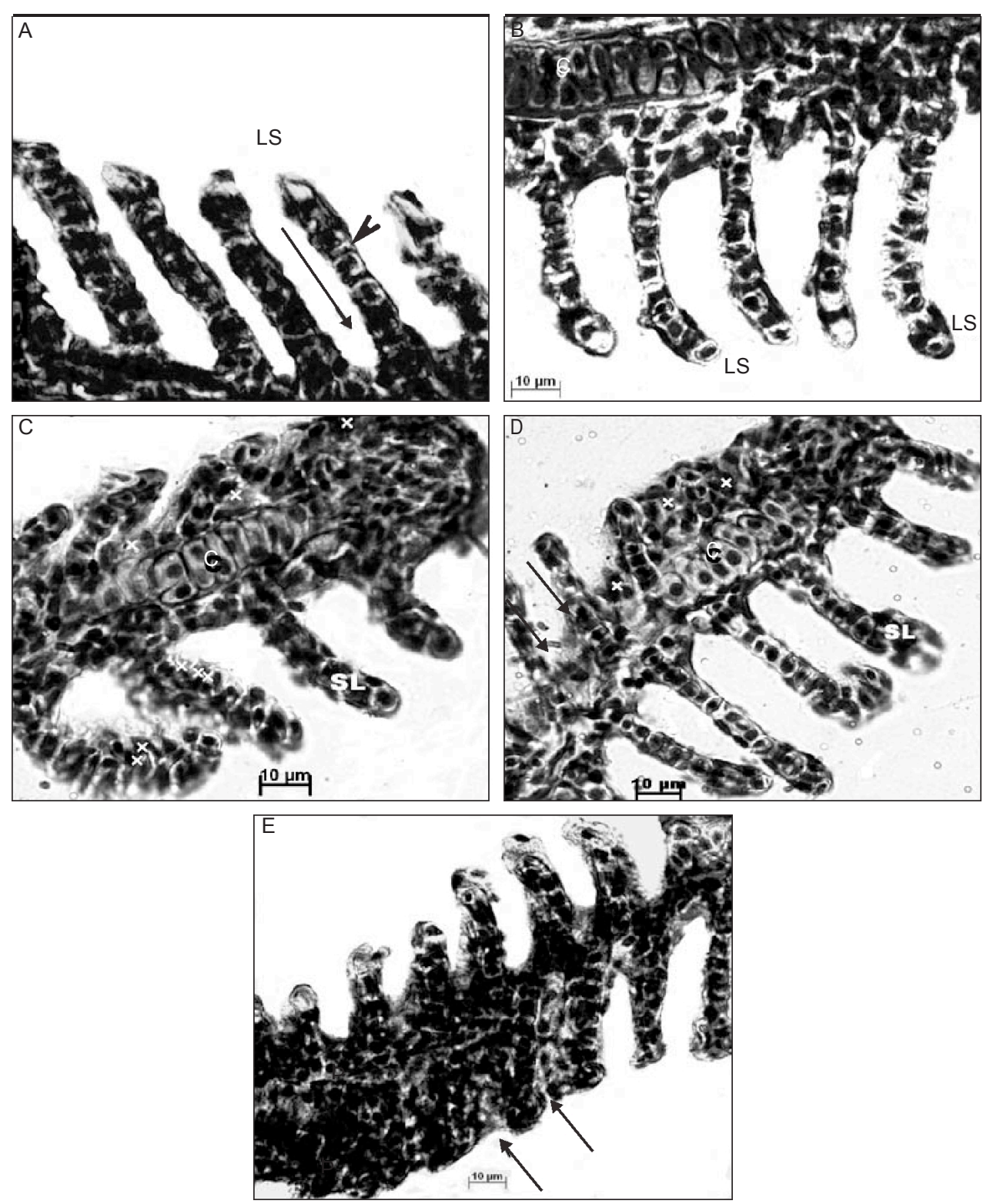

Figura 1 - Brânquias de D. rerio juvenil: (A) Controle: notar os espaços entre as lamelas secundárias (LS) bem definidos ( $)$ e o epitélio respiratório justaposto (今); (B) Controle + acetona: características semelhantes à do controle, com detalhe da cartilagem (letra c); (C) $0,003 \propto \mathrm{g} \mathrm{L}^{-1}$ : espessamento das lamelas secundárias (X) com a diminuição dos espaços entre elas; (D) $0,3 \propto \mathrm{g} \mathrm{L}^{-1}$ : espessamento das lamelas secundárias, principalmente no ápice das lamelas secundárias (SL), e fusão de algumas lamelas secundárias (X); (E) $3 \propto \mathrm{g} \mathrm{L}^{-1}$ : proliferação do tecido epitelial entre as lamelas secundárias (D) e a conseqüente fusão de algumas lamelas secundárias (1D). Aumento: 40x, bar: $10 \propto \mathrm{m}$ e coloração HE.

\section{CONCLUSÕES}

O endosulfan sulfato foi tóxico para os juvenis de $D$. rerio em concentrações ambientalmente relevantes. Este fato foi evidenciado pelas menores porcentagens de sobreviventes nos testes em relação ao lote controle e por meio das alterações histológicas detectadas nas brânquias, as quais foram pro- gressivas com o aumento das concentrações dos testes de toxicidade crônica parciais. Além disso, é importante destacar que, em ambientes naturais, as alterações podem ser mais acentuadas e comprometedoras para as populações, uma vez que estão sujeitas a exposição contínua desta e a outras substâncias químicas em concentrações mínimas nos ecossis-temas aquáticos. 
Agradecimentos - À FAPESP, pelo apoio financeiro, Processo $\mathrm{n}^{\mathrm{o}}$ 02/ 10494-6. À Dra Maria Olímpia, do Departamento de Química Ambien-tal, da Universidade de São Paulo, São Carlos, SP, pelas análises químicas de endosulfan sulfato.

\section{REFERÊNCIAS BIBLIOGRÁFICAS}

ASSOCIAÇÃO BRASILEIRA DE NORMAS TÉCNICAS, 2003, Projeto 00:001.44-001. Ecotoxicologia aquática: Toxicidade aguda - Método de ensaio com peixes. Rio de Janeiro, RJ.

ASSOCIAÇÃO BRASILEIRA DE NORMAS TÉCNICAS, 2007, ABNT NBR 15499. Ecotoxicologia Aquática - Toxicidade crônica de curta duração - Método de ensaios com peixes. Rio de Janeiro, RJ, 21p.

ALLAN, R. J., 2002, Os pesticidas orgânicos em meio ambiente aquático, com ênfase em sua origem e destino nos Grandes Lagos. In: S. Matsui, B. F. D. Barrett \& J. Banerjee (eds.). Diretrizes para o gerenciamento de lagos vol. 4: gerenciamento de substâncias tóxicas em lagos e reservatórios. Instituto Internacional de Ecologia, SP.

ANTONIUS, G. F. \& BYERS, M. E., 1997, Fate and movement of endosulfan under field conditions. Environmental Toxicology and Chemistry, 16(4): 644-649.

AYRES, M., AYRES Jr., M., AYRES, D. L. \& SANTOS, A. A., 2001, Bio Estat 2.0. Computacional Program.

BURATINI, S. V \& BRANDELLI, A., 2006, Bioacumulação. In: P. A. Zagatto \& E. Bertoletti (eds.). Ecotoxicologia Aquática - Princípios e Aplicações. RiMa Editora, São Carlos, SP, p. 55-88.

CAMPAGNA, A. F., ÉLER, M. N., FRACÁCIO, R., RODRIGUES, B. K. \& FENERICH-VERANI, N., 2007, The toxic potential of aldrin and heptachlor on Danio rerio juveniles (Cypriniformes, Cyprinidae). Ecotoxicology, 16: 289-298.

CONSELHO NACIONAL DO MEIO AMBIENTE, 2005, Resolução CONAMA 357, 17 de março de 2005.

DOMITROVIC, H. A., 2001, Toxicidad y respuesta histopatológica em Aequidens ortalegrensis (Pisces, Cichlidae) expuestos a endosulfan em ensayos de toxicidad aguda y em ensayos subletales.

DORES, E. F. G. C., DE-LAMONICA-FREIRE, E. M., 1999, Contaminação do ambiente aquático por pesticidas: via de contaminação e dinâmica dos pesticidas no ambiente aquático. Revista de Ecotoxicologia e Meio Ambiente: Pesticidas, 9: 1-18.

GORMLEY, K. L. \& TEATHER, K. L., 2003, Developmental, behavioral, and reproductive effects experienced by Japanese medaka (Oryziaslatipes) in response to short-term exposure to endosulfan. Ecotoxicology and Environmental Safety, 54: 330-338.

HAMILTON, M. A., RUSSO, R. C. \& THURSTON, R. U., 1977, Trimmed Sperman-Karber method for estimating median lethal concentration in toxicity bioassays. Environmental Science and Technology, 7: 714-719.

JONSSON, C. M., TOLEDO, M. C. F. \& ALVES, V. A. F., 1992, Alterações histopatológicas em duas espécies de teleósteos expostos ao inseticida endosulfan. Revista Brasileira deToxicologia, 5: 9-12.

JONSSON, C. M. \& TOLEDO M. C. F., 1993, Acute toxicity of endosulfan to the fish Hyphessobrycon bifasciatus and Brachydanio rerio. Archieves of Environmental Contamination and Toxicology, 24: 151-155.

MAAK, G. \& SEGNER, H., 2003, Morphological development of the gonads in zebrafish. Journal of Fish Biology, 62: 895-906.

NOWAK, B. \& SUNDERAN, R. I. M., 1991, Toxicity and bioaccumulation of endosulfan to mosquitofish, Gambusia affinis (Baird \& Girard).

Verh. Internat. Verein. Limnol., 24: 2327-2329.
OWENS, K. D. \& BAER, K. N., 2000, Modifications of the tropical Japanese medaka (Oryzia latipes) embryo larval assay for assessing developmental toxicity of Pentacholophenol and p,p'Dichlorodophenyltricholoethane. Ecotoxicology and Environmental Safety, 47: 87-95.

PELAÉZ-RODRIGUES, M., 2001, Avaliação da qualidade da água da bacia do Alto Jacaré- Guaçu / SP (Ribeirão do Feijão e Rio do Monjolinho) através de variáveis fisicas, químicas e biológicas. Tese de Doutorado, Escola de Engenharia de São Carlos, Universidade de São Paulo, São Carlos.

POLEKSIC, V. \& MITROVIC-TUTUNDIZIC, V., 1994, Fish gills as monitor of sublethal and

chronic effects of pollution. In: R. Muller \& R. Lloyd (eds.). Sublethal and Chronic effects of Pollutants on freshwater fish. Fishing News Books.

RAO, D. M. R. \& MURTY, A. S., 1982, Toxicity and metabolism of the endosulfan in three freshwater catfishes. Environmental Pollution (series A), 27: 223-231.

RAND, G. M \& PETROCELLI, S. R., 1985, Fundamentals of aquatic toxicology: methods and application. Hemisphere Publishing Corporation, London.

RASHATWAR, S. S. \& ILYAS, R., 1984, Effect of phosphomidon in a freshwater teleost fish, Nemachelius demisonii (Day) Histopathological and biochemical studies. Journal of Environmental Biology, 5(1): 1-8.

ROEX, E. W. M., VRIES, E. \& VAN GESTEL, C. A. M., 2002, Sensitivy of the zebrafish (Danio rerio) early life stage test for compounds with different models of action. Environmental Pollution, 120: 355-362.

SECRETARIA ESPECIAL DO MEIO AMBIENTE (SEMA), 1988, Avalia-ção da toxicidade crônica para peixes, 9p. In: Manual de testes para a avaliação da ecotoxicidade de agentes químicos. Brasília, DF.

SILVA, D. M. L., 2000, Avaliação dos compostos organoclorados na bacia do rio Piracicaba. Dissertação de Mestrado, Centro de Energia Nuclear na Agricultura, Universidade de São Paulo, Piracicaba.

SUNDERAM, R. I. M., CHENG, D. M. H. \& THOMPSON, G. B., 1992, Toxicity of endosulfan to native and introduced fish in Australia. Environmental Toxicology and Chemistry, 11: 1469-1476.

SWARUP, P. A., RAO, D. M. \& MURTY, A. S., 1981, Toxicity of endosulfan to the freshwater fish Cirrhinus mrigala. Bulletin of Environmental Contamination and Toxicology, 27: 850-855.

TAKASHIMA, F. \& HIBIA, T., 1995, An atlas of fish histology - normal and pathological features. Second Edition. Kodansha LTD., Tokyo, Japan.

UNITED STATES ENVIRONMETAL PROTECTION AGENCY, 1995, Quality criteria for water. Washington, DC.

WAN, T. M., KUO, J., BUDAY, C., SCHROOEDER, G., AGGELN, G. V. $\&$ PASTERNAK, J., 2005, Toxicity of $\alpha-, \beta-,(\alpha+\beta)$-endosulfan and their formulated and degradation products to Daphnia magna, Hyalela azteca, Onchorhynchus mykiss, Onchorhynchus kisutch, and biological implications in streams. Environmental Toxicology Chemistry, 24(5): 1146-1154. 